\title{
Quando e come dovrebbero essere sottoposti a screening per tumori carcinoidi timici e bronchiali i pazienti affetti da neoplasie endocrine multiple di tipo 1?
}

\author{
Roberta Modica $^{1}$ • Annamaria Colao ${ }^{1}$ - Antongiulio Faggiano ${ }^{1}$
}

Pubblicato online: 12 agosto 2016

C) Springer International Publishing AG 2016

Commento a:

When and how should patients with multiple endocrine neoplasia type 1 be screened for thymic and bronchial carcinoid tumours?

\section{N.S. Ospina, S. Maraka, V. Montori, G.B. Thompson, W.F. Young Jr. \\ Clin Endocrinol (2016) 84:13-16}

La Multiple Endocrine Neoplasia Type 1 (MEN 1) è una rara patologia ereditaria, a trasmissione autosomica dominante, determinata da una mutazione sul cromosoma 11 che predispone alla formazione di tumori, più comunemente a carico delle paratiroidi e dell'ipofisi (adenomi) e tumori neuroendocrini. La manifestazione clinica più frequente è l'iperparatiroidismo primario. Anche se i carcinoidi timici e bronchiali sono le manifestazioni meno comuni della MEN1, con una prevalenza rispettivamente del $2-8,2 \%$ e del 4,7$13,3 \%$, la loro associazione con un incremento di mortalità ha determinato un crescente interesse verso la loro diagnosi. Le linee guida della Endocrine Society raccomandano lo screening per carcinoidi timici e bronchiali con tomografia computerizzata (TC) o risonanza magnetica (RM) del torace ogni 1-2 anni. Tuttavia, la qualità delle evidenze su cui si basa questa raccomandazione è molto bassa [1].

Questo studio ha analizzato i dati della letteratura circa la presentazione clinica dei pazienti con carcinoidi timici e bronchiali. Questi si basano su studi osservazionali, la maggior parte dei quali retrospettivi, con un piccolo numero di pazienti inclusi (in totale 101 carcinoidi timici e 43 bronchiali) e, soprattutto, nessuno studio rivolto alla valutazione dell'efficacia dell'intervento diagnostico. La revisione della

$凶$ A. Faggiano

afaggian@unina.it

1 Dipartimento di Medicina Clinica e Chirurgia, Università "Federico II" di Napoli, Via Sergio Pansini 5, 80131 Napoli, Italia letteratura suggerisce che i carcinoidi timici sono associati a incremento di mortalità e sono causa di morte nel 19-21\% di pazienti con MEN1. Per i carcinoidi bronchiali, sebbene non sia riportato un incremento della mortalità, sussiste il dubbio di errata valutazione del rischio. Per entrambe le patologie, la sopravvivenza è migliorata dall'intervento chirurgico, quindi è importante la loro identificazione precoce. Nei due studi prospettici valutati è suggerito, inoltre, che l'implementazione delle strategie di screening determinerebbe un incremento dell'identificazione dei casi, anche se non è chiaro se ciò determinerebbe anche un miglioramento della prognosi.

Le sfide principali per ottenere evidenze cliniche a basso rischio di bias in pazienti con MEN1 riguardano la rarità della patologia e la valutazione dell'efficacia delle strategie di screening sugli outcome. Importanti benefici riguarderebbero il miglioramento della sopravvivenza e della qualità di vita; potenziali danni potrebbero essere i risultati falsi positivi, l'identificazione di noduli polmonari non correlati a MEN1, l'incrementata esposizione a radiazioni, l'ansia, i lunghi periodi di follow-up e i costi elevati.

Le evidenze disponibili a supporto delle attuali strategie di screening per i carcinoidi timici e bronchiali nella MEN1 presentano un rischio di bias moderato-alto. Sono pertanto necessari studi multicentrici randomizzati per valutare l'efficacia delle diverse strategie diagnostiche, le modalità e l'intervallo di tempo dell'intervento. Al momento si raccomanda una valutazione iniziale con TC o RM, seguita nel follow-up da alternanza di TC e RM, con un intervallo variabile in base al rischio, che va discusso attentamente con il paziente.

\section{Bibliografia}

1. Thakker RV, Newey PJ, Walls GV et al (2012) Endocrine Society. Clinical practice guidelines for multiple endocrine neoplasia type 1 (MEN1). J Clin Endocrinol Metab 97(9):2990-3011 